Aim A narrative literature review was undertaken with the aim of capturing understandings around choice and perceived risks in end-of-life care.

Methods Medline (Ebsco), PschINFO, Assia (Applied Social Sciences Index and Abstracts), British Nursing Index, High Wire Press, CINAHL (Current Index to Nursing and Allied Health Literature) were searched using the terms decision*, risk*, hospice and palliative care, end of life care. Hand searching of key texts also revealed relevant papers. Inclusion criteria included adult focus ( $>18 \mathrm{yrs}$ ), and UK focus only. Two hundred and forty two papers were examined for relevance and filtered using criteria outlined by Hawker, et al (2002). In total, twenty five papers were analysed for key themes.

Results Notions of 'choice' are shaped by individual understandings as well as wider structural factors. Individual understandings include perceptions of services, family support, and balancing of self-identity. Structural factors include service provision, professional awareness, and inequalities around ethnicity and class.

Conclusions Acknowledgement by key stakeholders of the structural factors, which constrain choice, may help to inform service development. At the same time, recognition of patient and carer understandings may help to facilitate supportive decision making at end-of-life.

\section{P 036 HOW DO PERCEPTIONS OF RISK SHAPE 'CHOICE' IN END-OF-LIFE CARE?}

Fiona Wilson, ${ }^{1}$ Christine Ingleton, ${ }^{2}$ Merryn Gott, ${ }^{3}$ Clare Gardiner ${ }^{4} .{ }^{1}$ Sheffield Hallam University, Sheffield, UK; ${ }^{2}$ School of Nursing \& Midwifery University of Sheffield, UK; ${ }^{3}$ Faculty of Medical \& Health Science University of Auckland NZ; ${ }^{4}$ School of Nursing, Faculty of Medical \& Health Science University of Auckland NZ

10.1136/bmjspcare-2014-000654.77

Background The National End of Life Care Strategy for England (2008) advocates 'choice' as a central tenet of palliative care delivery, however it is not clear how 'choice' is understood nor how choice may be shaped by perceptions of risk. 